\title{
Electron Transport Characteristics of Wurtzite GaN
}

\author{
F. M. Abou El-Ela and A. Z. Mohamed \\ Department of Physics, Faculty of Girls, Ain Shams University, Heliopolis, Cairo 11757, Egypt \\ Correspondence should be addressed to F. M. Abou El-Ela; fadlaeg@yahoo.com
}

Received 18 June 2013; Accepted 16 July 2013

Academic Editors: Y. Ohta, R. Rossmanith, and S. Wang

Copyright (C) 2013 F. M. Abou El-Ela and A. Z. Mohamed. This is an open access article distributed under the Creative Commons Attribution License, which permits unrestricted use, distribution, and reproduction in any medium, provided the original work is properly cited.

A three-valley Monte Carlo simulation approach was used to investigate electron transport in wurtzite GaN such as the drift velocity, the drift mobility, the average electron energy, energy relaxation time, and momentum relaxation time at high electric fields. The simulation accounted for polar optical phonon, acoustic phonon, piezoelectric, intervalley scattering, and Ridley charged impurity scattering model. For the steady-state transport, the drift velocity against electric field showed a negative differential resistance of a peak value of $2.9 \times 10^{5} \mathrm{~m} / \mathrm{s}$ at a critical electric field strength $180 \times 10^{5} \mathrm{~V} / \mathrm{m}$. The electron drift velocity relaxes to the saturation value of $1.5 \times 10^{5} \mathrm{~m} / \mathrm{s}$ at very high electric fields. The electron velocities against time over wide range of electric fields are reported.

\section{Introduction}

In recent years, nearly all electronic and optoelectronic devices have been realized using alloys of the III-V nitrides, gallium nitride $(\mathrm{GaN})$, aluminum nitride $(\mathrm{AlN})$, and indium nitride $(\mathrm{InN})[1,2]$. There has been considerable interest in $\mathrm{GaN}$ due to its wide band gap and favorable material properties, such as high electron mobility and very high thermal conductivity [3]; GaN and related compounds with aluminum and indium currently have great potential for applications in optoelectronics devices in the frequency range from microwaves to ultraviolet, quantum dots infrared photodetectors [4-7]. GaN also has large speed electron velocity and can be used for electronics devices [8].

The electron transport characteristics of the III-V nitride semiconductor, GaN, have long been recognized. In 1975, Littlejohn et al. [9] were the first to report results obtained from semiclassical Monte Carlo simulations of the steadystate electron transport within bulk wurtzite GaN.

In 1993, Gelmont et al. [10] reported on ensemble semiclassical two-valley Monte Carlo simulations of the electron transport within bulk wurtzite GaN; this analysis improvs upon the analysis of Littlejohn et al. [9], by incorporating intervalley scattering into the simulations. In 1995, Mansour et al. [11] reported the use of such an approach in order to determine how the crystal temperature influences the velocity-field characteristic associated with bulk wurtzite
GaN. Kolnik et al. [12] reported on employing full-band Monte Carlo simulations of the electron transport within bulk wurtzite GaN and bulk zinc-blende GaN.

In 1998, Albrecht et al. [13] reported on employing ensemble semiclassical five-valley Monte Carlo simulations of the electron transport within bulk wurtzite $\mathrm{GaN}$, with the aim of determining elementary analytical expressions for a number of electron transport parameters corresponding to bulk wurtzite GaN. The first known study of transient electron transport within the III-V nitride semiconductors was that carried by Foutz et al. reported in 1997 [14].

On the experimental front, in 2000, Wraback et al. [15] reported on the use of a femtosecond optically detected timeof-flight experimental technique in order to experimentally determine the velocity-field characteristic associated with bulk wurtzite GaN.

An understanding of the electron transport in $\mathrm{GaN}$ is necessary to improve $\mathrm{GaN}$ semiconductors based devices. Transport properties of GaN at both steady state and transient state has been discussed extensively over the year; uncertainty in material band parameters remains a key source of ambiguity in analysis of the electron transport properties.

\section{Monte Carlo Simulation}

In order to calculate the electron drift velocity for large electric fields, consideration of conduction band satellite 
TABLE 1: The material parameters corresponding to wurtzite GaN.

\begin{tabular}{lc}
\hline Parameter & Value \\
\hline Mass density $\left(\mathrm{kg} / \mathrm{m}^{3}\right)$ & 6150 \\
Sound velocity $(\mathrm{m} / \mathrm{s})$ & 6560 \\
Acoustic deformation potential $(\mathrm{eV})$ & 8.3 \\
Static dielectric constant & 8.9 \\
High-frequency dielectric constant & 5.35 \\
Piezoelectric constant, $e_{14}\left(\mathrm{C} / \mathrm{m}^{2}\right)$ & 0.375 \\
Optical phonon energy $(\mathrm{eV})$ & 0.0912 \\
Intervalley deformation potentials $(\mathrm{eV} / \mathrm{m})$ & $10^{11}$ \\
Intervalley phonon energies $(\mathrm{eV})$ & 0.0912 \\
\hline
\end{tabular}

TABLE 2: The valley parameters corresponding to wurtzite GaN.

\begin{tabular}{lccc}
\hline Valley & $\Gamma_{1}$ & $\Gamma_{2}$ & $\mathrm{~L}-\mathrm{M}$ \\
\hline Valley degeneracy & 1 & 1 & 6 \\
Effective mass & $0.2 m_{e}$ & $m_{e}$ & $m_{e}$ \\
Intervalley energy separation $(\mathrm{eV})$ & - & 1.9 & 2.1 \\
Energy gap $(\mathrm{eV})$ & 3.39 & 5.29 & 5.49 \\
Nonparabolicity $\left(\mathrm{eV}^{-1}\right)$ & 0.189 & 0.0 & 0.0 \\
\hline
\end{tabular}

valleys is necessary. A three-valley model of the conduction band using parameters for wurtzite phase $\mathrm{GaN}$ is used. The scatterings considered in our calculation are polar optical phonon, acoustic deformation potential, piezoelectric, charged impurity scattering, and intervalley scattering. Our Monte Carlo simulations differ from other monte carlo simulation due to the use of Ridley charged impurity model and including the electron temperature at high electric fields. For more details of these scattering mechanisms, the reader is referred to [16-19].

We simulate 10000 electrons and the crystal temperature is set to $300 \mathrm{~K}$. Nonparabolicity is considered in the lowest conduction band valley; this nonparabolicity is treated through the application of the Kane model [17-19].

The material parameters used for our simulations are tabulated in Table 1. These parameters selections are from [1] and originally from [20] for GaN. The valley parameters selections are presented in Table 2 [1]. These parameters were originally determined from the band structural calculations of [21].

\section{Charged Ionized Impurity Scattering Rate}

The scattering rate of electron by charged ionized donor in GaN may be calculated according to Brooks and Herring (B.H) model [22]

$$
\Gamma_{\mathrm{BH}}(k)=\frac{\sqrt{2} N_{I} e^{4} m^{* 1 / 2}}{8 \varepsilon^{2} \hbar^{2} q_{c}^{2}} \frac{(1+2 \alpha E)}{\gamma^{1 / 2}(E)}\left[\frac{1}{1+\left(q_{c} / 2 k\right)^{2}}\right] .
$$

$E$ represents the electron energy, $m^{*}$ is the effective mass, $\alpha$ is the nonparabolicity factor, and $\varepsilon$ represents the high frequency dielectric constant, where $N_{I}$ is the density of impurity atoms, while $q_{c}$ is the inverse screening length

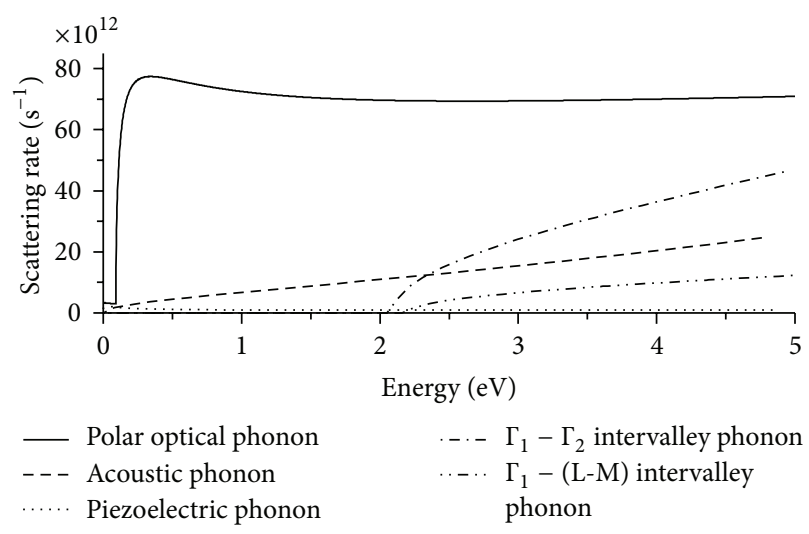

FIGURE 1: Scattering rates versus electron energy in the $\Gamma_{1}$ valley.

defined by $q_{c}=\sqrt{N e^{2} / \varepsilon k_{B} T_{e}}, N$ is the electron concentration, $T_{e}$ is the electron temperature, and $\gamma(E)=E(1+\alpha E)$.

Ridley $[16,19]$ proposed a model, which becomes convenient to use in the Monte Carlo method [23] since it solves the problem of cutting the impurity scattering at very small electron energy in the B.H model, at the same time it reconciles between the different proposed models used before

$$
\Gamma_{R}(k)=\frac{v(k)}{a}\left[1-\exp \left(-\frac{a \Gamma_{\mathrm{BH}}(k)}{v(k)}\right)\right]
$$

$\Gamma_{R}(k), \Gamma_{\mathrm{BH}}(k)$ represent the Ridley and the Brooks Herring impurity scattering rates respectively; $v(k)$ is the electron group velocity and $a$ is the average distance apart the impurity center which is given by Ridley $[16,17]$

$$
a=\left(2 \pi N_{I}\right)^{-1 / 3} \text {. }
$$

\section{Results and Discussion}

Figure 1 illustrates the computed scattering rates for phonons in the $\Gamma_{1}$ valley; it is clear that piezoelectric scattering rate weakens towards high electron energies. The principle source of scattering is that due to optical type phonons either through polar interaction or through intervalley deformation potential mechanism. It is seen that the acoustic scattering rate is very small compared with polar optical phonon scattering rate.

Figure 2 shows a comparison between the Brooks Herring (B.H) model at ionized impurity concentration of $10^{17} \mathrm{~cm}^{-3}$ and the Ridley model $(R)$, at different impurity concentration. In both models we have assumed the electron temperature equivalence $300 \mathrm{~K}$. Additionally, the impurity concentration is taken to be equivalent to the free electron concentration. There is a large reduction of the scattering rate in the Ridley $(R)$ model compared to the (B.H) model, especially at small electron energy. Consequently, it is more reasonable to use the Ridley model in the Monte Carlo method, since it solves the problem of cutting the impurity scattering at very small electron energy. 


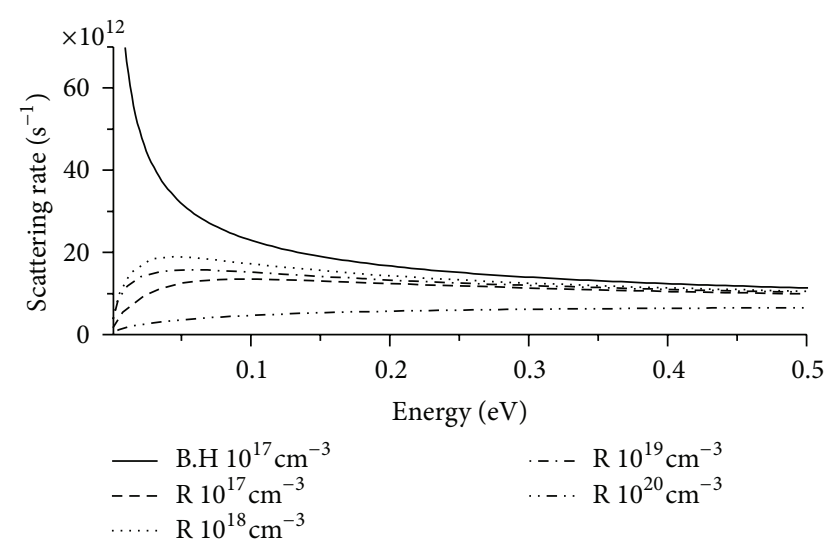

FIGURE 2: Impurity scattering rates versus electron energy in the $\Gamma_{1}$ valley.

The velocity-field characteristic associated with Wurtzite $\mathrm{GaN}$ is presented in Figure 3 as function of electric fields for lattice temperature $300 \mathrm{~K}$ and ionized impurity concentration equal to $10^{17} \mathrm{~cm}^{-3}$; results from other group and various available experimental data [24-26] are also included for comparison.

At applied electric fields less than $50 \times 10^{5} \mathrm{~V} / \mathrm{m}$, the scattering processes are dominated by acoustic deformation potential, piezoelectric phonon scattering, and impurity scattering. Thus, at low fields, where the electron temperature is less than the polar optical phonon temperature, Polar optical phonon emissions are a rare event and the free carriers remain a majority in the $\Gamma_{1}$ valley where their effective mass is weak. Their speed increases with the electric field to reach a maximum value of $2.9 \times 10^{5} \mathrm{~m} / \mathrm{s}$ for a critical field $180 \times$ $10^{5} \mathrm{~V} / \mathrm{m}$.

At the critical field, the electrons gain more and more energy so they are excited and transferred to the upper valleys where they become heavier. The possibility of their collisions increases, which slows them and their steady-state velocity begins to reduce. This is in response to further increases in the applied electric field strength; that is, a region of negative differential mobility is observed. At very high fields, the electron saturation drift velocities are in the order of $1.5 \times$ $10^{5} \mathrm{~m} / \mathrm{s}$. Our calculations of steady-state drift velocity curve were in a fair agreement with those given by other groups at low electric field while at high field experimental values are significantly lower. Also due to the scattering mechanisms used and different chosen data parameters there are modest agreement at high fields with other simulation results.

The average electron energy as a function of the applied electric field is shown in Figure 4, initially, the average electron energy remains low, only slightly higher than the thermal energy $3 / 2 k_{B} T$. We see that there is a sudden increase around $150 \times 10^{5} \mathrm{~V} / \mathrm{m}$. This increase is due to the fact that the dominant energy loss mechanism for $\mathrm{GaN}$ is polar optical phonon scattering. When the applied electric field is less than $100 \times 10^{5} \mathrm{~V} / \mathrm{m}$, all of the energy that the electrons gain from the applied electric field is lost through polar optical phonon scattering. The other scattering mechanisms, that

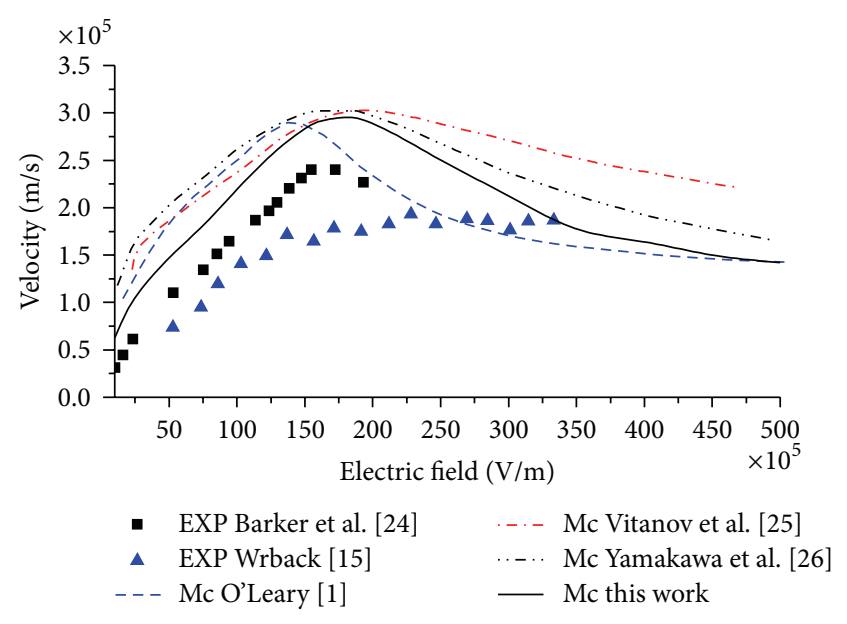

FIgURE 3: The velocity-field characteristic.

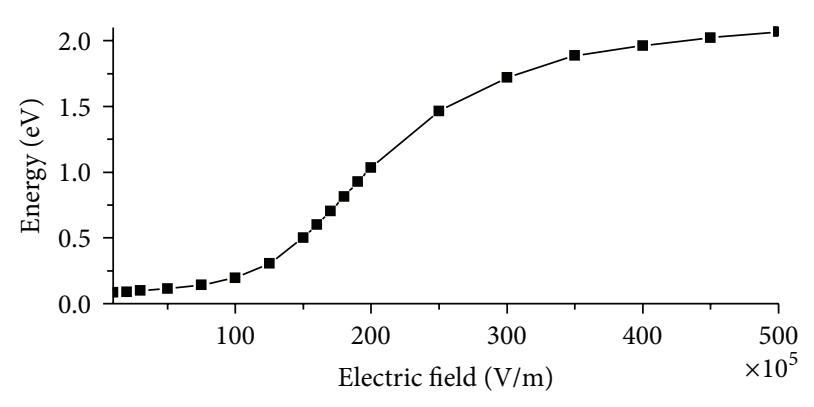

FIGURE 4: The average electron energy as a function of the applied electric field.

is, piezoelectric scattering, impurity scattering, and acoustic deformation potential scattering, do not remove energy from the electron ensemble; they are elastic scattering mechanisms.

However, beyond certain critical applied electric field strength, the polar optical phonon scattering mechanism can no longer remove all of the energy gained from the applied electric field. Other scattering mechanisms must start to play a role if the electron ensemble is to remain in equilibrium. The average electron energy increases until intervalley scattering begins and an energy balance is reestablished.

Figure 5 illustrates the fractional electron number as a function of the applied electric field. As the applied electric field is increased beyond $150 \times 10^{5} \mathrm{~V} / \mathrm{m}$, the average electron energy increases until a substantial fraction of the electrons have acquired enough energy in order to transfer into the upper valleys of the conduction band. As the effective mass of the electrons in the upper valleys is greater than that in the lowest valley, the electrons in the upper valleys will be slower. Finally, at high applied electric fields, the number of electrons in each valley saturates. At this point, the electron drift velocity stops decreasing and achieves saturation.

The spreading of the distribution function (D.F.) as a function of energy in the $\Gamma_{1}$ valley of $\mathrm{GaN}$ is shown in Figure 6, where the electric field varies from $50 \times 10^{5} \mathrm{~V} / \mathrm{m}$ to $300 \times 10^{5} \mathrm{~V} / \mathrm{m}$. The kink in the energy distribution appears at electron energy equivalent to the phonon energy, that is, 


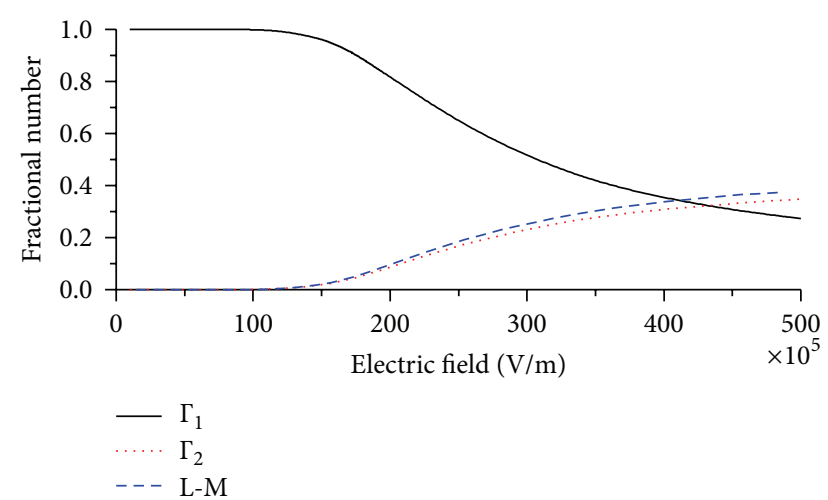

FIGURE 5: The fractional electron number as a function of the applied electric field.

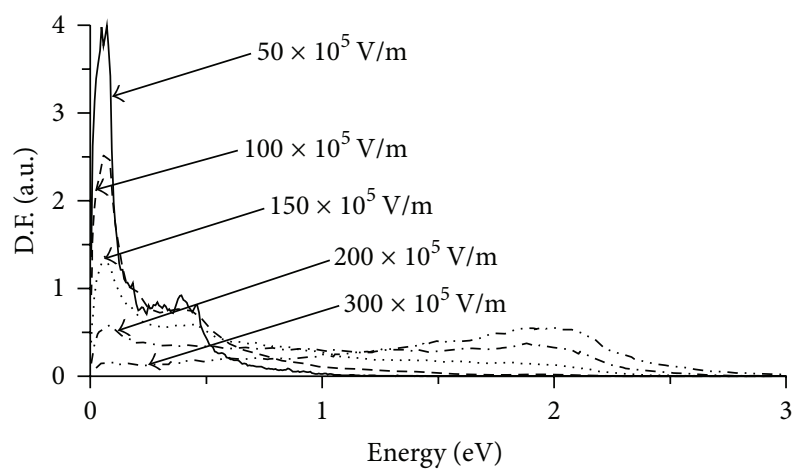

FIGURE 6: The number of distribution functions against electron energy in the $\Gamma_{1}$ valley.

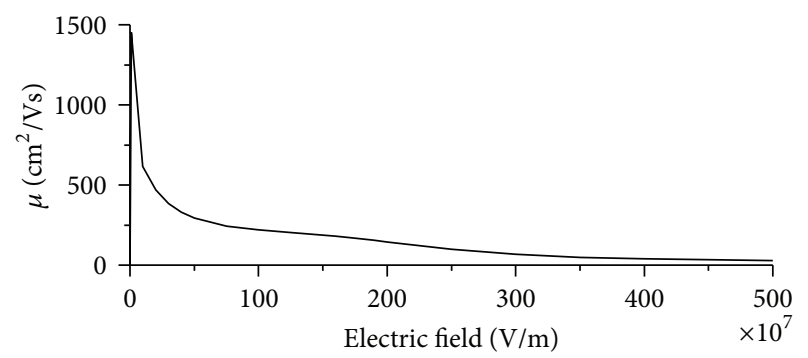

FIGURE 7: The average drifts mobility as a function of electric field.

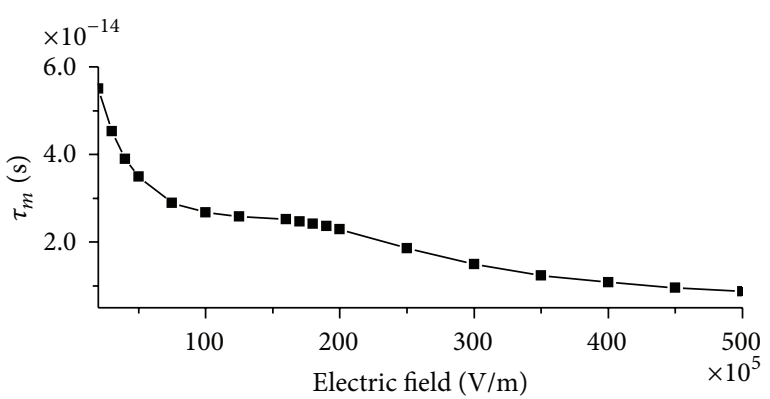

FIGURE 8: The momentum relaxation time as a function of the electric field.

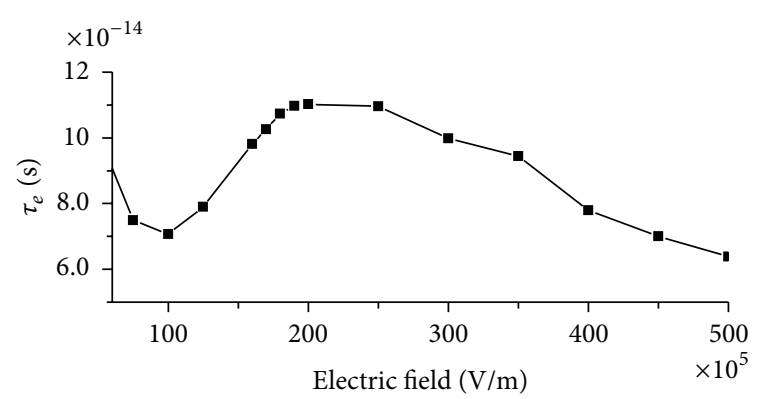

FIGURE 9: The Energy relaxation time as a function of the electric field.

$\hbar \omega$. There is another kink when the electron energy reaches $2 \hbar \omega$, represents iterative emission of two phonons, which is possible only at lattice temperature of $300 \mathrm{~K}$.

In Figure 7, the average electron drift mobility has its maximum value at very low values of the electric field due to the smallest effective mass for the electrons in the central valley. The negative differential mobility NDM is observed by increasing the electric field due to intervalley mechanism. Linear low-field electrons drift velocity dependence on the applied electric field, $v_{d}=\mu F$ in this regime. At high electric field, the average electron drift mobility reaches low values due to the increase of electron effective mass in higher valleys.

The effective momentum relaxation time $\tau_{m}$ and the effective energy relaxation time $\tau_{e}$ at steady state are given respectively, by Foutz et al. [20]:

$$
\tau_{m}=\frac{m^{*} V_{d}}{q F}, \quad \tau_{e}=\frac{E-E_{0}}{q V_{d} F},
$$

where $E_{0}=3 / 2 K_{B} T_{L}, T_{L}$ is the lattice temperature, and $K_{B}$ is Boltzmann constant. The energy-dependent effective mass $m^{*}$ is an average of the effective mass $\left(m^{*}=m_{0}^{*}(1+2 \alpha E)\right)$ over the ensemble.

Figures 8 and 9 show the momentum relaxation time and the energy relaxation time versus electric fields in GaN. The momentum relaxation time decreases as electric field increases at applied electric fields corresponding to a field higher than $100 \times 10^{5} \mathrm{~V} / \mathrm{m}$, which is a negative differential resistance (NDR) region due to intervalley scattering to higher $\Gamma_{2}$ and $\mathrm{M}-\mathrm{L}$ valleys. On the contrary, the energy relaxation time increases for the same reasons which were already mentioned.

We examine transient electron transport in bulk wurtzite $\mathrm{GaN}$ for a variety of applied field strengths. Figure 10 illustrates the time dependence of the electron drift velocity and the electron energy for electric field ranging between $(50-300) \times 10^{5} \mathrm{~V} / \mathrm{m}$. Below the critical field $\left(170 \times 10^{5} \mathrm{~V} / \mathrm{m}\right)$, the electron drift velocity reaches steady state very quickly with no velocity overshoot. For applied electric field strengths above the critical value, the electrons gain a greater energy in the $\Gamma_{1}$ valley where the electron effective mass is lowest. They are getting a great acceleration which enables them to reach the electron peak velocity; that is, an overshoot occurs. Then the electrons transfer to the upper valleys where they become 


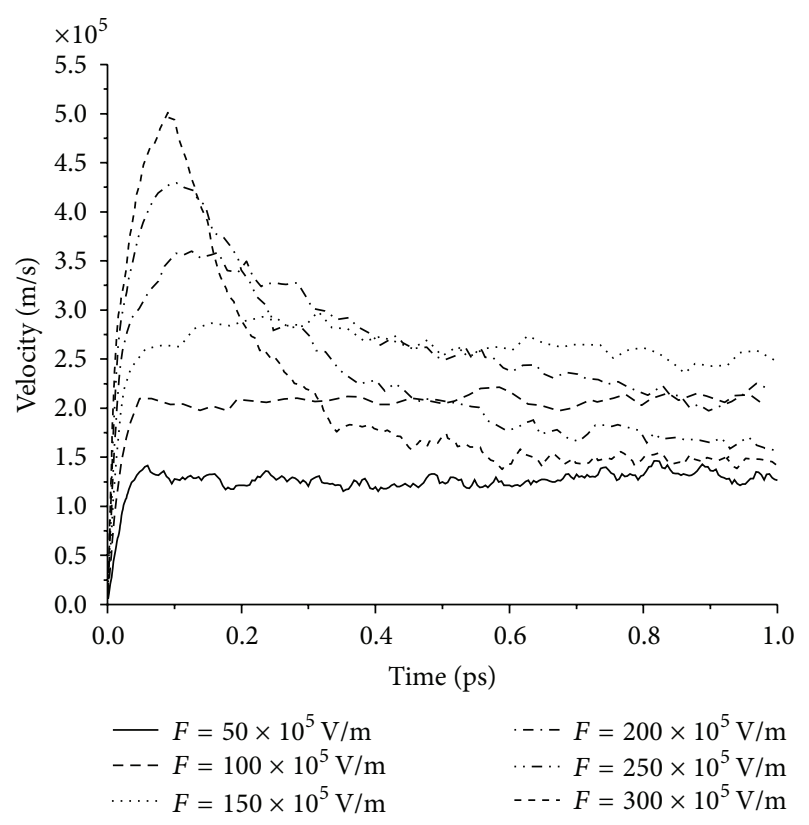

(a)

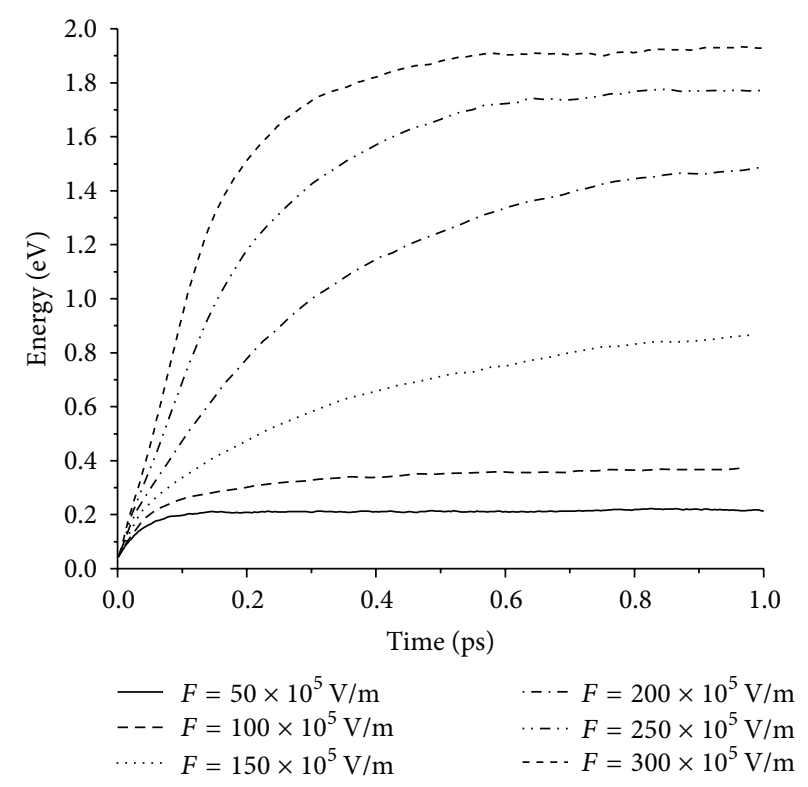

(b)

FIGURE 10: The time dependence of (a) the electron drift velocity and (b) the electron energy.

heavier and their velocity decreases toward its steady-state value.

\section{Conclusions}

Electron transport in wurtzite $\mathrm{GaN}$ at room temperature has been simulated by the ensemble Monte Carlo method. The main feature of our calculated results, due to the scattering mechanisms, is an ohmic region at low fields lower than the threshold field, where all electrons occupy the $\Gamma_{1}$ valley, followed by a negative differential resistance, which occurs as a consequence of the intervalley electron transfer mainly from the $\Gamma_{1}$ to the $\Gamma_{2}$ valley at fields larger than the threshold field, since a significant number of electrons can have energies in excess of the $\Gamma_{1}-\Gamma_{2}$ difference and it is possible for them to be scattered in the $\Gamma_{2}$ valley by short wave length phonons. An electron in the $\Gamma_{2}$ valley has an effective mass much larger than its value in the $\Gamma_{1}$ valley. Consequently, the average velocity becomes less than the ohmic value as the field strength increases. The transfer to the $\Gamma_{2}$ minimum is sufficiently rapid as the field strength increases; thus, the intervalley transfer mechanism could lead to a negative differential mobility. Finally, the velocity overshoot occurs only over very small distances for electrons injected at higher energies.

\section{References}

[1] S. K. O.'Leary, B. E. Foutz, M. S. Shur, and L. F. Eastman, "Steady-state and transient electron transport within the III-V nitride semiconductors, GaN, AlN, and InN: a review," Journal of Materials Science, vol. 17, no. 2, pp. 87-126, 2006.
[2] V. M. Polyakov and F. Schwierz, "Low-field electron mobility in wurtzite InN," Applied Physics Letters, vol. 88, no. 3, Article ID 032101, p. 3, 2006.

[3] U. V. Bhapkar and M. S. Shur, "Monte Carlo calculation of velocity-field characteristics of wurtzite GaN," Journal of Applied Physics, vol. 82, no. 4, pp. 1649-1655, 1997.

[4] S. Chen and G. Wang, "High-field properties of carrier transport in bulk wurtzite GaN: a Monte Carlo perspective," Journal of Applied Physics, vol. 103, no. 2, Article ID 023703, 2008.

[5] A. Dyson and B. K. Ridley, "High-field transport and terahertz generation in GaN," Journal of Applied Physics, vol. 104, no. 11, Article ID 113709, 2008.

[6] K. H. Lee, P. C. Chang, S. J. Chang, C. L. Yu, Y. C. Wang, and $\mathrm{S}$. L. Wu, "GaN MSM photodetectors with an unactivated Mgdoped GaN cap layer and sputtered ITO electrodes," Journal of the Electrochemical Society, vol. 155, no. 6, pp. J165-J167, 2008.

[7] S. V. Averine, P. I. Kuznetzov, V. A. Zhitov, and N. V. Alkeev, "Solar-blind MSM-photodetectors based on $\mathrm{Al}_{x} \mathrm{Ga}_{1-x} \mathrm{~N} / \mathrm{GaN}$ heterostructures grown by MOCVD," Solid-State Electronics, vol. 52, no. 5, pp. 618-624, 2008.

[8] E. Monyor, F. Omnés, and F. Calle, "Wide-bandgap semiconductor ultraviolet photodetectors," Semiconductor Science and Technology, vol. 18, no. 4, pp. R33-R51, 2003.

[9] M. A. Littlejohn, J. R. Hauser, and T. H. Glisson, "Monte Carlo calculation of the velocity-field relationship for gallium nitride," Applied Physics Letters, vol. 26, no. 11, pp. 625-627, 1975.

[10] B. Gelmont, K. Kim, and M. Shur, "Monte Carlo simulation of electron transport in gallium nitride," Journal of Applied Physics, vol. 74, no. 3, pp. 1818-1821, 1993.

[11] N. S. Mansour, K. W. Kim, and M. A. Littlejohn, "Theoretical study of electron transport in gallium nitride," Journal of Applied Physics, vol. 77, no. 6, pp. 2834-2836, 1995.

[12] J. Kolnik, I. H. Oğuzman, K. F. Brennan, R. Wang, P. P. Ruden, and Y. Wang, "Electronic transport studies of bulk zincblende 
and wurtzite phases of GaN based on an ensemble Monte Carlo calculation including a full zone band structure," Journal of Applied Physics, vol. 78, no. 2, Article ID 1033, 1995.

[13] J. D. Albrecht, R. P. Wang, P. P. Ruden, M. Farahman, and K. F. Brennan, "Monte Carlo calculation of electron transport properties of bulk AIN," Journal of Applied Physics, vol. 83, no. 3, Article ID 1446, 1998.

[14] B. E. Foutz, S. K. O'Leary, M. S. Shur, and L. F. Eastman, "Comparison of high field electron transport in GaN and GaAs," Journal of Applied Physics Letter, vol. 70, no. 21, Article ID 2849, 1997.

[15] M. Wraback, H. Shen, J. C. Carrano et al., "Time-resolved electroabsorption measurement of the electron velocity-field characteristic in GaN," Applied Physics Letters, vol. 76, no. 9, pp. 1155-1157, 2000.

[16] B. K. Ridley, "Reconciliation of the Conwell-Weisskopf and Brooks-Herring formulae for charged-impurity scattering in semiconductors: third-body interference," Journal of Physics C: Solid State Physics, vol. 10, no. 10, pp. 1589-1593, 1977.

[17] B. K. Ridley, Quantum Processes in Semiconductors, Claredon Press, Oxford, UK, 1988.

[18] C. Jacoboni and L. Reggiani, "The Monte Carlo method for the solution of charge transport in semiconductors with applications to covalent materials," Reviews of Modern Physics, vol. 55, pp. 645-705, 1983.

[19] C. Jacoboni and P. Lugli, The Monte Carlo Method For SemiconDuctor Device Simulation, Springer, Vienna, Austria, 1989.

[20] B. E. Foutz, S. K. O’ Leary, M. S. Shur, and L. F. Eastman, "Transient electron transport in wurtzite GaN, InN, and AlN," Journal of Applied Physics, vol. 85, no. 11, Article ID 7727, 8 pages, 1999.

[21] W. R. L. Lambrecht, B. Segall, and J. H. Edgar, Properties of Group III Nitrides, EMIS Data reviews Series, chapter 4, INSPEC, London, UK, 1994.

[22] H. Brooks and C. Herring, "Scattering by Ionized Impurities in Semiconductors," Physical Review, vol. 83, pp. 879-887, 1951.

[23] T. G. van de Roer and F. P. Widdershoven, "Ionized impurity scattering in Monte Carlo calculations," Journal of Applied Physics, vol. 59, no. 3, pp. 813-815, 1986.

[24] J. M. Barker, R. Akis, D. K. Ferry et al., "High-field transport studies of GaN," Physica B, vol. 314, no. 1-4, pp. 39-41, 2002.

[25] S. Vitanov, M. Nedjalkov, and V. Palankovski, "A Monte Carlo model of piezoelectric scattering in GaN," in Proceedings of the 6th international conference on Numerical methods and applications (NMA '06), vol. 197, Springer, Berlin, Germany, 2007.

[26] S. Yamakawa, S. Aboud, M. Saraniti, and S. Goodnick, "Influence of the electron-phonon interaction on electron transport in wurtzite GaN," Semiconductor Science and Technology, vol. 19, no. 4, p. S475, 2004. 

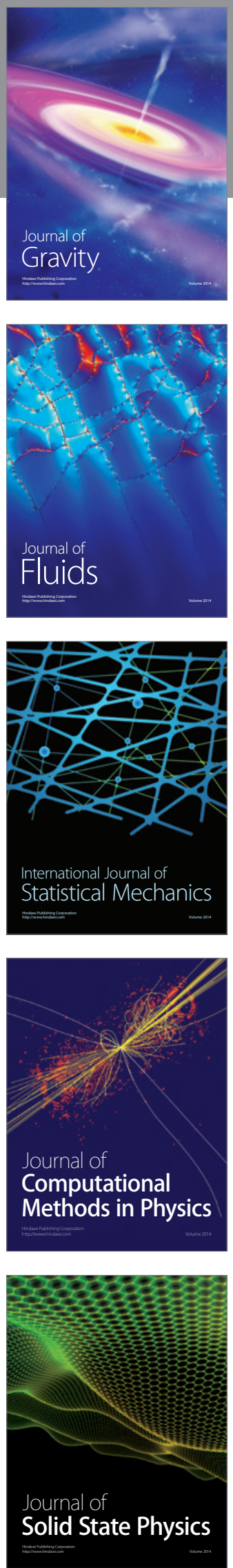

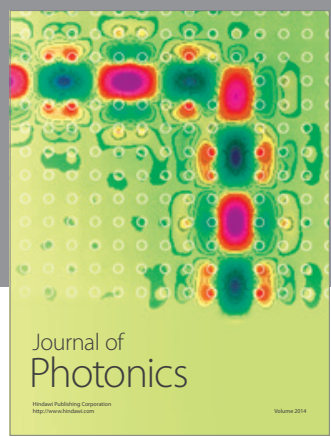

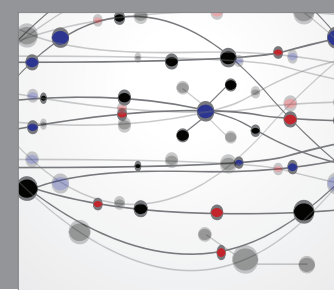

The Scientific World Journal

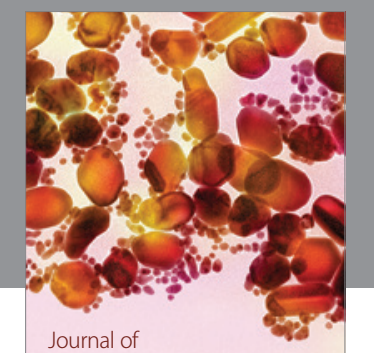

Soft Matter
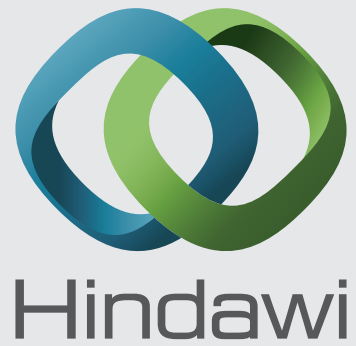

Submit your manuscripts at

http://www.hindawi.com
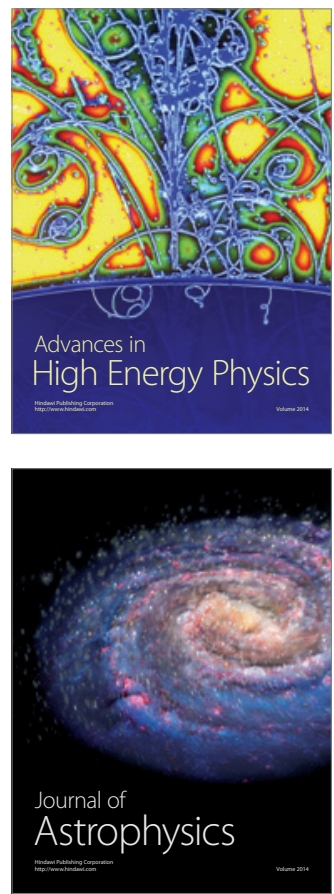
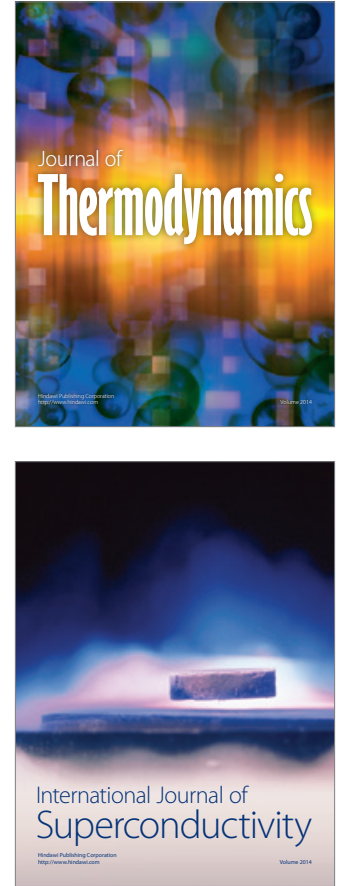
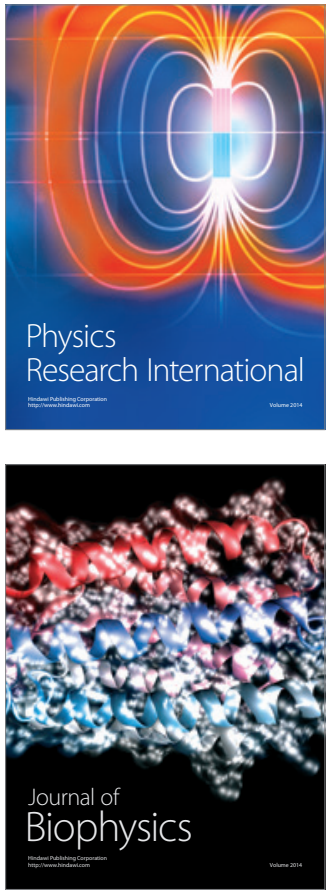
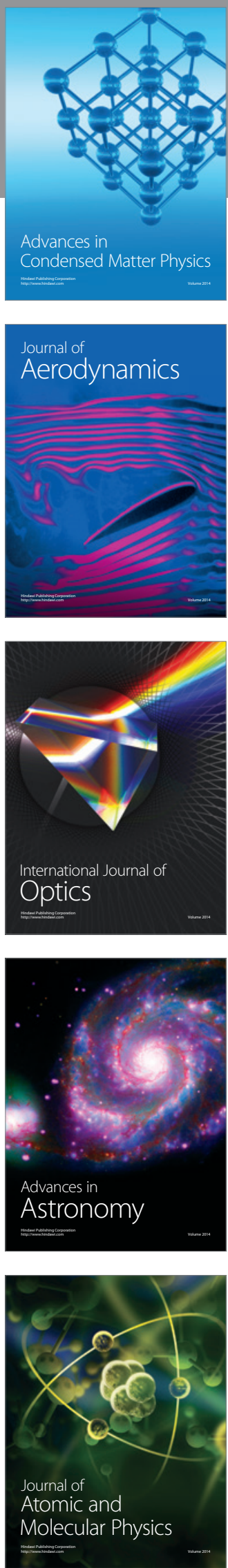